\title{
Localized left ventricular myocardial defect leading to fatal cerebral embolism
}

\author{
G. R. Fitzgerald, W. J. K. Cumming, D. J. Scott, D. S. Reid, and A. S. Hunter \\ From the Departments of Cardiology and Pathology, Newcastle General Hospital, Newcastle upon Tyne
}

The case of a previously healthy I7-year-old youth who presented with a fatal cerebral infarct is described. $A$ localized defect in the anterior left ventricular myocardium with overlying thrombus, and left ventricular hypertrophy were found at necropsy. An old infarct was found in the left kidney. There were no signs of ischaemic or inflammatory heart disease. The possible origins of the lesion are discussed.

Systemic embolism from the heart in young people is a rarity in the absence of rheumatic heart disease, infective endocarditis, or premature ischaemic disease, and is associated with uncommon types of heart disease, e.g. ventricular aneurysms (Chesler, Tucker, and Barlow, 1967; Pocock et al., 1965), tumours (Joynt, Zimmerman, and Khalifeh, I965), penetrating injuries (Killen et al., 1969), and cardiomyopathy (Harvey, Segal, and Gurel, 1964). This communication describes a fatal cerebral embolism in an apparently healthy I7-year-old youth with a small localized defect in the left ventricular muscle.

\section{Case report}

The patient was admitted to the neurosurgical unit of the Newcastle General Hospital because of right hemiplegia with aphasia of sudden onset. There was no history of trauma or evidence of external injury. Skull $x$ ray and cerebrospinal fluid analysis were normal. $\mathrm{He}$ was referred to the cardiology department because of an irregular pulse and cardiac enlargement. A history taken from his parents and family doctor revealed that he had been a healthy young man who had regularly engaged in energetic outdoor sports until the day of admission. $\mathrm{He}$ had not suffered from any serious illnesses and there had not been any symptoms of rheumatic fever or of cardiac dysfunction. On examination he was a welldeveloped young man who appeared healthy apart from an obvious right hemiplegia with motor aphasia. He was alert and co-operative. There was no clubbing or cyanosis. Pulse rate was 67 per minute with frequent extrasystoles. The blood pressure was $130 / 80 \mathrm{mmHg}$ (17.3)
I0.6 $\mathrm{kPa}$ ) in both arms. Femoral pulses were normal. Venous pressure was normal. The cardiac impulse was easily visible and palpable outside the left midclavicular line and was sustained and heaving in character. The right ventricle was not palpable and there were no thrills. A faint midsystolic murmur was audible at the left sternal border. Apical third and fourth heart sounds

\section{D.McK. 7yrs $25 \cdot 2 \cdot 74$}
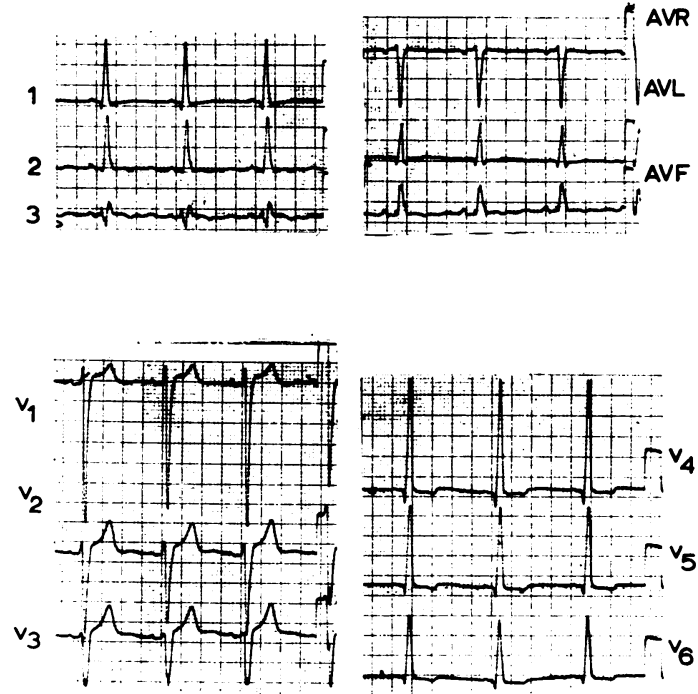

FIG. I The electrocardiogram on admission. For description, see text. 
were heard. The pulmonary component of the second sound was accentuated. Lung bases were clear. Peripheral pulses, ocular fundi, and thyroid gland were normal. Neurological examination confirmed the presence of a right-sided flaccid hemiplegia and motor aphasia. Physical examination was otherwise normal.

\section{Investigations}

Blood: erythrocyte sedimentation rate $3 \mathrm{~mm} / \mathrm{hr}$, haemoglobin $15.1 \mathrm{~g} / \mathrm{dl}$, white cell count ro $300 / \mu \mathrm{l}$ (neutrophils $90 \%$, lymphocytes 10\%, no eosinophils seen), SGOT I4 units $/ \mathrm{ml}$. Urea and electrolytes normal.

Electrocardiogram: (Fig. I) sinus rhythm, normal AV conduction, QRS width 0.1 I to $0.12 \mathrm{~s}$, voltage $\mathrm{SV2}+$ $R_{5}=39 \mathrm{~mm}, T$ wave inversion in II, III, aVF, $V_{4}$ to V6; flat $T$ in lead I. Low $R$ wave voltage VI to $V_{3}(R V I=$ $5 \mathrm{~mm}, \mathrm{RV}_{2}=3 \mathrm{~mm}, \mathrm{RV}_{3}=\mathrm{I} \mathrm{mm}$ ).

Chest X-ray: cardiothoracic ratio $\mathrm{I5} / 24.5$ (6I \%). Probable left ventricular enlargement.

Phonocardiogram: confirmed the presence of third and fourth heart sounds, and systolic murmur.

Echocardiogram: Normal mitral and aortic valve movement. Normal septal thickness. No anterior systolic movement of anterior mitral leaflet. Ejection fraction = 0.53 .

Mean rate of circumferential fibre shortening (Vcf) $0.69 \mathrm{circs} / \mathrm{s}$ (normal = I.29 \pm 0.23 ) (Cooper et al., 1972).

\section{Clinical progress}

The patient gradually deteriorated over the subsequent 48 hours, developing signs of increasing cerebral oedema despite parenteral administration of corticosteroids and mannitol. He developed cardiorespiratory arrest on the fourth hospital day and attempted resuscitation was unsuccessful. A necropsy was performed I2 hours post mortem.

\section{Necropsy findings}

The major findings were in the brain and heart. The skull and dura were normal. The brain weighed $1440 \mathrm{~g}$. Thère was a prominent left tentorial pressure cone and the left cerebral hemisphere appeared swollen, with flattening of the convolutions and haemorrhages into the cortex. Circle of Willis and internal carotid arteries were normal and no major occlusions were seen. On section there was extensive recent infarction in the territory of the left middle cerebral artery. This focally haemorrhagic infarction involved the cortex of the frontal and parietal lobes and adjacent white matter.

The heart weighed $420 \mathrm{~g}$. Systemic venous drainage and right atrium were normal, and pulmonary valve was normal. The right ventricular cavity was normal with a wall thickness up to $3 \mathrm{~mm}$. Pulmonary veins, left atrium, and mitral valve were normal. The left ventricle was hypertrophied and dilated, the myocardial thickness measuring up to $18 \mathrm{~mm}$. Near the apex there were two

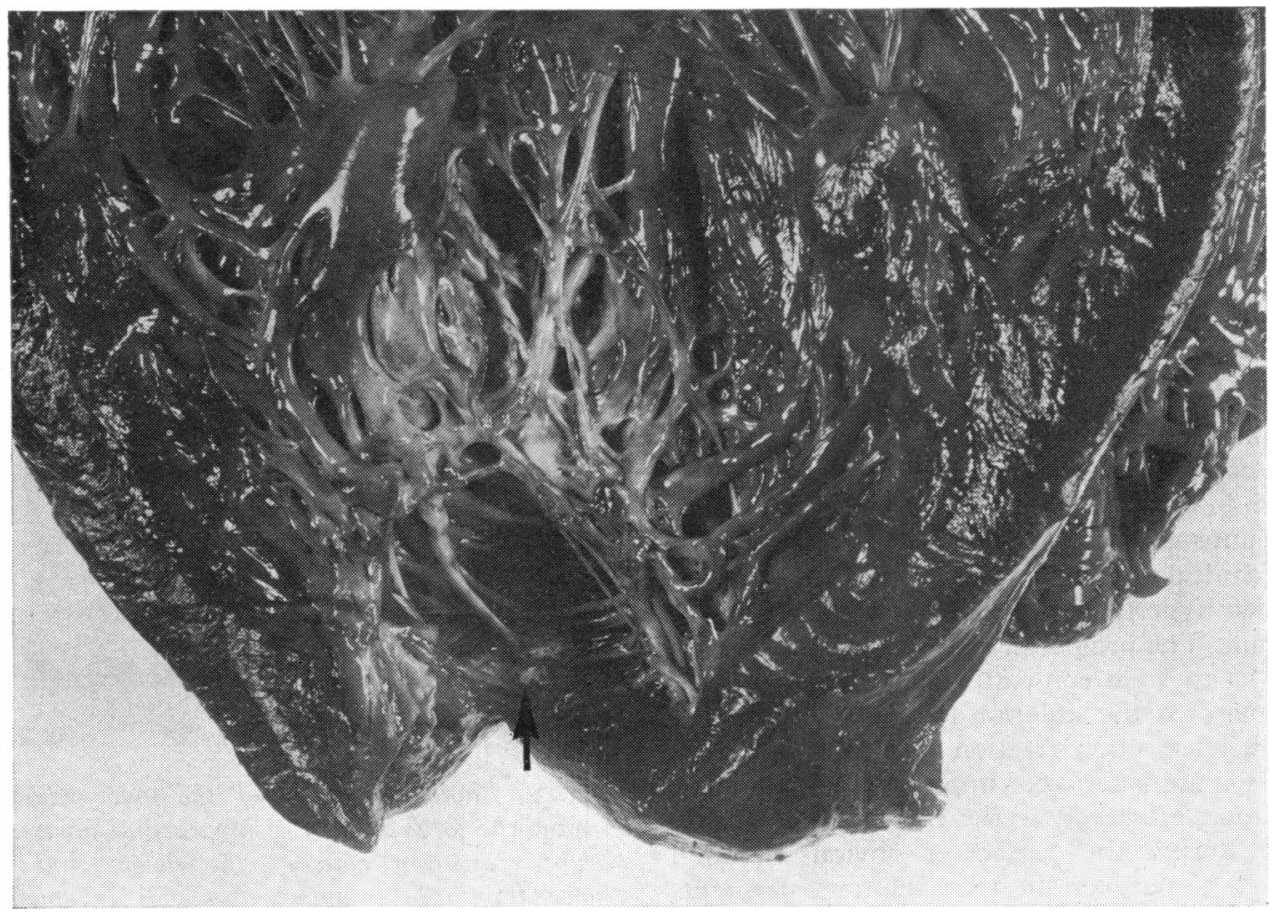

FIG. 2 Internal aspect of the left ventricle showing the myocardial defect (arrow). 
areas ( 15 and $10 \mathrm{~mm}$ in diameter) where the myocardium appeared to be absent, leaving a thin membrane $2 \mathrm{~mm}$ in thickness (Fig. 2). On the internal aspect, these areas were overlaid by a complex network of malformed, thin trabeculae, many of which showed subendocardial fibrosis. Thrombus was enmeshed between these trabeculae. The remainder of the left ventricle appeared normal as did the aortic valve. The coronary ostia were patent and the coronary artery distributions were normal. The internal surfaces of the coronary arteries were normal and no occlusions were found in any of the major branches.

The left kidney weighed $130 \mathrm{~g}$. There was a depressed scar on the cortex measuring $15 \times 7 \times 5 \mathrm{~mm}$. The capsule was adherent in this area.

No other gross abnormalities were present.

\section{Microscopical examination}

Brain The presence of recent focal haemorrhagic infarction was confirmed.

Heart Sections were stained with haematoxylin and eosin and Weigert's elastic stain (Hart's modification). There was focal replacement of the myocardium of part of the anterior wall of the left ventricle by dense acellular fibrous tissue (Fig. 3). There was an abrupt transition between the normal myocardium and the defect, where the endocardium and epicardium were separated by a thin layer of fibrous tissue containing only a few elastic fibres, but no myocardial fibres. The adjacent myocardium was normal, but there was slight thickening of the elastic and connective tissue. There was no inflammatory reaction and no haemosiderin.

Sections from multiple areas of the left ventricle, left atrium, mitral valve, right ventricle, and coronary arteries showed no abnormality.

Sections from the left kidney showed a wedge-shaped area of scarring with tubular atrophy, many hyalinized glomeruli, and a lymphocytic infiltrate, appearances consistent with previous infarction.

\section{Discussion}

The important and interesting features of this case are the unheralded and subsequently fatal cerebral embolism in a previously healthy active young man, and the unusual cardiac findings at necropsy. The cardiac clinical features were mainly those of left ventricular hypertrophy confirmed by the electrocardiogram. The progressive loss of $R$ wave voltage in leads $V_{1}$ to $V_{3}$ is compatible with a reduction in muscle mass in the anterior left ventricular wall. The reduced ejection fraction and Vcf shown by echocardiography indicated impaired left ventricular performance. However, as the echocardiogram was recorded during the period of obvious cerebral oedema it is not certain that the measurements would be unaffected by intracranial factors. The necropsy confirms a focal cerebral infarction and a most likely single source of embolus in the left ventricle. An old left kidney scar, compatible with previous infarction, may indicate a previous systemic embolus. The lesion in the left ventricular wall is characterized by focal defects in the myocardium of the anterior wall without evidence of extensive scarring or inflammatory reaction. The possible causes of this lesion are those discussed by Paranetto and Strauss (1963) in their presentation of an 8-month-old girl with a left ventricular aneurysm. Though aneurysmal dilatation was not present in our case, the myocardial defect without flbrous or inflammatory reaction is very similar. In the absence of any evidence to suggest coronary artery disease or previous inflammatory disease, they concluded that the lesion was probably a developmental abnormality. Ischaemic heart disease in our case is unlikely because of the findings of corenary arteries

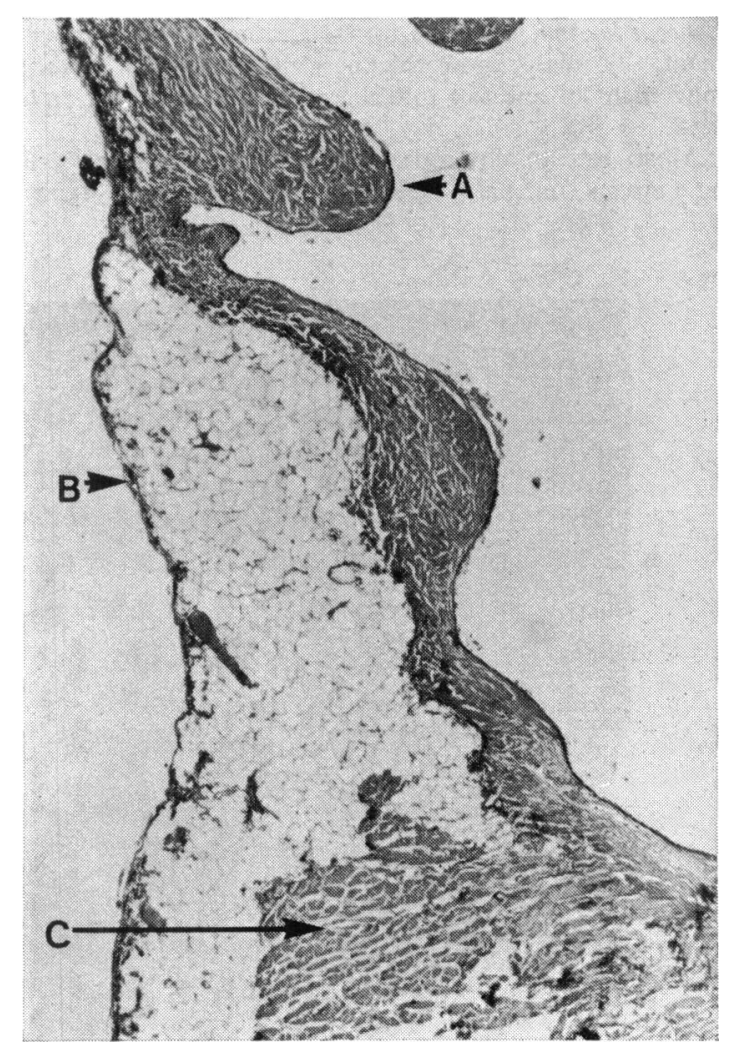

FIG. 3 Photomicrograph of the myocardial defect to show the total absence of myocardial fibres and the abrupt transition between the defect and normal myocardium. $A$ is endocardium; $B$ is epicardium; $C$ is normal myocardium. (Weigert's elastic stain. $\times$ 39.) 
with normal origin, size, and distribution which are without occlusion or narrowing and which show no evidence of inflammatory or degenerative arterial disease. Furthermore, there is no demonstrable source of coronary emboli apart from the left ventricular lesion itself. The absence of inflammatory or fibrotic changes in the lesion or the rest of the left ventricle is evidence against a previous inflammatory condition being responsible for the lesion. Therefore, the possibility of a developmental abnormality is more likely. Congenital aplasia or absence of the myocardium has been described by Uhl (1952) and several further cases have been reported since (Diaz et al., 1973). These cases demonstrate a primary congenital defect, with abnormal development of the primordium of the right ventricular myocardium. The same morphological and histological appearance in our patient may indicate a similar abnormality occurring in the left heart. The reason for left ventricular hypertrophy in our case is obscure, but possibilities include undetected previous arterial hypertension and hypertrophy compensatory for the myocardial defect, as a result of disordered left ventricular contraction.

\section{References}

Chesler, E., Tucker, R. B. K., and Barlow, J. B. (1967). Subvalvular and apical left ventricular aneurysms in the Bantu as a source of systemic emboli. Circulation, 35, I 156.
Cooper, R. H., O'Rourke, R. A., Karliner, J. S., Peterson, K. L., and Leopold, G. R. (1972). Comparison of ultrasound and cineangiographic measurements of the mean rate of circumferential fiber shortening in man. Circulation, 46, 914 .

Diaz, L. P., Jiménez, M. Q., Granados, F. M., Martinez, V. P., and Batres, G. M. (1973). Congenital absence of myocardium of right ventricle. British Heart fournal, 35, 570.

Harvey, W. P., Segal, J. P., and Gurel, T. (1964). The clinical spectrum of primary myocardial disease. Progress in Cardiovascular Diseases, 7, I7.

Joynt, R. J., Zimmerman, G. E., and Khalifeh, R. (1965). Cerebral emboli from cardiac tumors. Archives of Neuro$\log y, 12,84$.

Killen, D. A., Gobbel, W. G., Jr., France, R., and Vix, V. A. (1969). Post-traumatic aneurysm of the left ventricle. Circulation, 39, 101.

Paronetto, F., and Strauss, L. (1963). Aneurysm of the left ventricle due to congenital muscle defect in an infant. American fournal of Cardiology, 12, 721 .

Pocock, W. A., Cockshott, W. P., Ball, P. J. A., and Steiner, R. E. (1965). Left ventricular aneurysms of uncertain aetiology. British Heart fournal, 27, 184 .

Uhl, H. S. M. (1952). A previously undescribed congenital malformation of heart: almost total absence of the myocardium of the right ventricle. Bulletin of the fohns Hopkins Hospital, 91, 197.

Requests for reprints to Dr. W. J. K. Cumming, Department of Medicine, Newcastle General Hospital, Westgate Road, Newcastle upon Tyne NE4 6BE. 